\title{
Research on the Application of Virtual Reality Technology in Psychotherapy
}

\author{
Lulu Liu ${ }^{1}$, Yan Liu, * \\ 1 School of Humanities, Jiangxi University of Traditional Chinese Medicine, Nanchang, Jiangxi, China
}

\begin{abstract}
The popularization and application of virtual reality technology provides a new way for psychotherapy. This paper expounds the advantages of virtual reality technology and its specific application in the treatment of psychological diseases such as social anxiety, phobia and eating disorder. At the same time, aiming at the psychological pressure problem faced by modern people, a set of psychological adjustment system for improving emotional experience and virtual scene is designed based on behaviourism-oriented psychotherapy theory and virtual reality technology.
\end{abstract}

\section{Introduction}

Psychotherapy, in a broad sense, refers to any technique and measure that can slow down or treat psychological, emotional or behavioural disorders. Traditional psychotherapy techniques, such as psychoanalytic therapy, behavioural therapy, humanistic psychotherapy, cognitive therapy, etc., play an important role in psychological counseling and treatment and have achieved fruitful results. However, the traditional psychotherapy technology inevitably has some limitations, such as: the treatment environment is single and it is difficult to restore the scene, relying too much on visitors to dictate their memories and experiences, which may lack authenticity. The development and application of virtual reality can make up for some shortcomings of traditional psychotherapy ${ }^{[1]}$.

Burdea, a pioneer in the field of virtual reality, believes that "virtual reality is a synthetic computer user interface that simulates reality through a variety of perceptual channels, such as sight, hearing, touch, smell, and so on." others think that virtual reality is a virtual threedimensional space generated by computer simulation, which enables users to interact with the virtual world immersively and triggers a series of events ${ }^{[2]}$. This technology has gradually developed from the initial stage of the lack of human-computer interaction to the flourishing period of commercialization and systematization. More and more people are aware of the broad prospects of this technology and apply it to different industries and fields, one of them includes the medical profession. Many researchers at home and abroad have applied this technology to the field of psychotherapy and evaluation, which undoubtedly brings convenience and good news to psychotherapy.

\section{Application Advantages of Virtual Reality Technology in Psychotherapy}

Virtual Reality technology is a new technology developed in recent 20 years, which mainly uses high-tech equipment such as computer, helmet display, data glove and so on to build a real-time interactive $3 \mathrm{D}$ virtual world for users. It is immersive, interactive and imaginative. When it is applied to the field of psychotherapy, it shows some advantages ${ }^{[3]}$ as follows:

\subsection{Security}

Virtual reality technology can simulate dangerous situations and significantly improve treatment conditions, and effectively ensure the safety of patients. Different from traditional therapy, virtual reality provides a safe environment for visitors to experience and explore boldly. In the course of treatment, visitors can explore and improve their skills in various mistakes, difficulties and even unpredictable events without any harm.

\subsection{Controllability}

Virtual reality is also controllable. It can not only design the treatment process and create a personalized treatment environment according to the actual situation of visitors and the degree of psychological barriers, but also better understand the characteristics of patients in memory, attention, imagination, emotional response, etc., so as to correctly diagnose its etiology and formulate corresponding treatment plans.

\subsection{Realistic Sense of Presence}

Virtual reality technology can provide visitors with

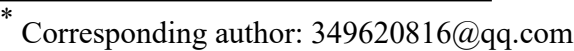


various intuitive sensory stimuli. In the virtual environment, they can see various scenes, hear different sounds, touch different objects and smell different tastes, thus intuitively feeling the environment and events. Realistic presence is conducive to the presentation of psychological problems of visitors and the improvement of treatment effect.

\subsection{Experiential Nature}

In the virtual scene, visitors can not only experience various situations in the real society, but also cross the boundaries of time and space, and experience scenes that can't appear in the real society, such as experiencing war or disaster, so as to gain wonderful insights and change personal cognition

\section{Application of Virtual Reality Technology in Psychotherapy}

\subsection{Treatment of Social Anxiety Disorder}

Virtual reality is used to make specific virtual scenarios and expose patients to them, thus causing anxiety and achieving the purpose of treating anxiety disorder. Slater et al. first applied VR technology to the study of social anxiety. They designed a virtual social scene, established a virtual character to engage in social activities with patients, and the patients were placed in the virtual social scene for treatment. In the virtual environment, the anxiety situation of patients is gradually exposed, and the anxiety threshold of patients is gradually raised, thus improving the symptoms of patients.

\subsection{Treatment of Phobia}

In the treatment of phobia, VR technology is mainly used to create a virtual situation that makes patients feel fear, and put patients in this virtual situation to implement exposure therapy [4]. In addition to using exposure therapy to treat acrophobia in virtual scenes, virtual reality technology can also be used to treat mental diseases such as flight phobia, claustrophobia and agoraphobia. Compared with the real situation, the use of virtual situation in the treatment process can prevent patients from appearing too strong fear reaction and ensure the personal safety of patients at the same time.

\subsection{Treatment of Emotional Distress}

In the form of combining VR technology with art therapy, the virtual reality helmet and the brush matched with the helmet are used to create, so that people can freely enter the "Painting world" to create art, and let patients release their anxiety, ease emotional conflicts and clarify past experiences through creation. At the same time, in the process of transforming ideas into concrete images, personal needs and emotions are conveyed, so that their personality can be adjusted and treated.

\subsection{Treatment of Eating Disorders}

Eating disorder is a mental disorder with abnormal eating behaviour. Most eating disorders are due to the misunderstanding of one's own body image. Riva [5] and others first combined VR technology with cognitive behavioural therapy to form experiential cognitive therapy, which was applied to the treatment of eating disorders. Their treatment mainly uses VR technology to create five virtual and distorted realistic environments and combines Socrates' questioning method in cognitive therapy to help patients have a more realistic cognition of their own body image. Using VR technology, researchers can not only correct patients' wrong body image cognition, but also deal with patients' evasive behaviour and negative emotions caused by food.

\subsection{Treatment of Schizophrenia}

The treatment of schizophrenia with VR technology is the research direction only in recent years, and the research results are still few, and it is still in the experimental stage of development. However, foreign researchers have used VR technology to treat schizophrenia patients with positive symptoms, and found that treatment can effectively alleviate the hallucinations and delusions of schizophrenia patients. In this way, VR technology can be used to make schizophrenic patients simulate the hallucinations in their minds and create an illusion avatar. Then let the patients face this illusion incarnation, and make them realize that their illusion is untrue and unreasonable, which is caused by morbid psychology. For those who can't get a radical cure, this method can help them adapt to their own hallucinations and improve their social function. The following is a sketch of the application of VR technology in specific psychological problems.

In the application of VR technology, in the face of different parties, we can flexibly use the theory and technology of psychotherapy schools. Behavioral therapy can use virtual situations to activate the psychological disorders of the parties, cognitive therapy can use VR technology to evaluate situational memory or disintegrate habitual selective attention patterns, psychoanalytic school therapy can use it as a complex symbol system to evoke or release emotions, expressive art therapy can use VR technology to build an artistic expression platform to ease emotional conflicts. Although VR technology can be widely used in the treatment of mental illness, it still needs the correct guidance of therapists. 


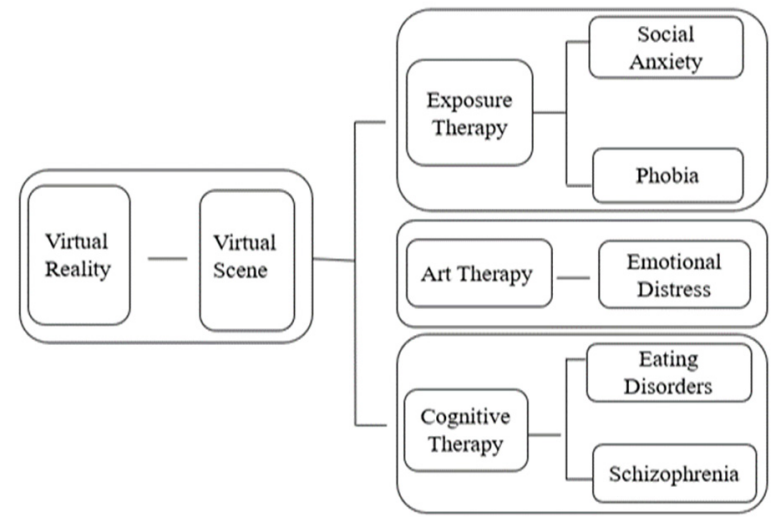

Fig 1. Application of VR technology in psychotherapy

\section{Design of Virtual Visual System for Psychotherapy Process}

\subsection{Design Ideas}

With the development of society, people's pace of life is accelerating, the competition for survival is becoming increasingly fierce, and interpersonal relationships are becoming more complex. More and more people feel strong psychological pressure, frequently appear negative emotions such as depression, anxiety, depression, sadness, and even produce psychological distress and psychological obstacles. This design is based on behaviorist relaxation therapy, combined with meditation technology, using virtual reality technology to build a three-dimensional virtual scene system suitable for meditation and relaxation. The system can effectively attract the attention of visitors, make them focus their attention on their feelings, experience a sense of relaxation, completely immerse themselves in meditation, relieve their inner troubles, release their emotions, and achieve the role of psychological intervention and adjustment ${ }^{[6]}$. Although the scene is virtual, the experience it gives people is real. The system can effectively attract the attention of visitors, make them pay attention to listen to their own physical feelings, experience a sense of relaxation, completely immerse themselves in meditation, relieve their inner troubles, release their emotions, and achieve the role of psychological intervention and adjustment.

\subsection{Frame Composition}

The system consists of two parts: software and hardware. the hardware part includes display stereo vision equipment helmet display, digital equipment, data gloves and input equipment keyboard and mouse. The hardware is connected with the software system through the computer interface. The software package consists of five parts, which is composed of environment, special effects, sound, prompts and system detection. It mainly provides three types of services, namely, mindfulness meditation, natural scenery and music relaxation ${ }^{[7]}$. The system framework is shown in figure 2. In a word, visual environment, auditory environment, active treatment experience and psychological adjustment hints work together to form a psychological regulation system suitable for relaxation and meditation therapy. Hardware data gloves, mouse keyboard and virtual reality helmet are used to realize the experience of virtual reality psychotherapy system, using sensing devices to achieve continuous man-machine information updates, see stereoscopic pictures, and hear simulated sounds. Achieve an immersive relaxation experience and get a complete three-dimensional relaxation meditation therapy experience.

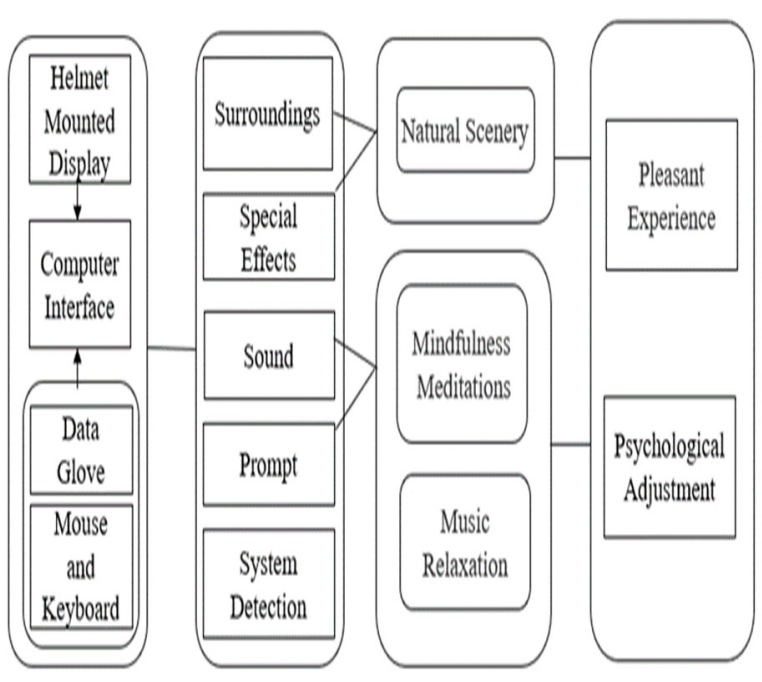

Fig 2. Design of VR psychological adjustment system

\subsection{Treatment Process}

The visitor sits on a very comfortable chair, sees the virtual scene directly through the virtual vision device, interacts with the scene through the interactive device data glove, mouse and keyboard, roams the virtual venue freely from his own point of view, browses, experiences and listens, and fully enjoys the beauty and interest. Moreover, the emotional responses and cognitive changes that occur in this process can be transferred to real life. If you have virtual reality equipment, you can experience extraordinary things at home, even if you don't leave the house. Virtual life, the use of computers and immersive three-dimensional helmet equipment, get immersive relaxation experience and meditation experience, release and relieve the psychological pressure from work, study, life and other aspects, make the mind and body happy and relaxed.

\section{Conclusion}

With the free ride of Internet technology, the development of mental health service industry is also changing with each passing day. Virtual reality technology has the advantages of immersion, interaction and imagination. The application of virtual reality technology in psychotherapy can not only improve the treatment 
conditions, ensure the safety and privacy of patients, but also facilitate therapists to manipulate the treatment process, and greatly improve the efficiency of treatment. However, the virtual reality system is only a standardized program, and its flexibility and pertinence are poor, so it can not be used for every visitor. And psychological counseling or therapy emphasizes the relationship between counselors and visitors. VR technology is only an auxiliary means but can not replace the role of counselors. In addition, it takes a long time to build a new virtual environment, and requires a high cost of research and development, so it can not be used for special phobia or some rare mental diseases. VR equipment is also expensive and needs regular maintenance, which will increase the cost of treatment for patients.

This paper mainly introduces the application and development trend of virtual reality technology abroad from the point of view of clinical practice, and on the basis of the full application of VR technology and the advantages and disadvantages in the field of psychology, this paper designs a virtual scene system for healthy people, which can be used in the process of psychotherapy, which can help visitors release negative emotions and relieve all kinds of stress, so as to realize the beauty of life with a healthy state of mind.

\section{References}

1. Wu Hao. (2016). VR/AR technology will break through human psychological perception and bring the ultimate image realistic experience. News Research Guide (19),335.

2. Ni Bin. (2018). Application of Virtual Reality Technology and VR Equipment in Psychological Intervention Therapy. Computer Products and Circulation (02), 168-169.

3. Li Jian. (2017). Research on the Application of VR Virtual Reality Technology in the Field of Psychological Rehabilitation.

4. Xiao Haichuan,Liao Ying,Che Jingshang. (2019). New progress in VR virtual reality technology and treatment of acrophobia. Electronic Technology and Software Engineering (02),119-121

5. Zhang Xiaoyu. (2017). Application of Virtual Reality Technology in Medicine. Technology and Innovation (03), 161 .

6. Fan Dongmei. (2019). Design and Implementation of Psychological Relaxation System Based on VR Technology. Overseas Chinese Garden (07),124.

7. Gu Chuhan. (2019). Application of Virtual Reality Technology in Medicine. Science and Technology Communication (06),129-130. 